\title{
Intervención educativa para la prevención de la escabiosis dirigida a representantes de preescolares de 5-6 años
}

\author{
Educational intervention for the prevention of scabiosis Aimed at representatives of \\ preschoolers of 5-6 years

\section{Intervenção educativa para a prevenção da escabiose dirigida a representantes de crianças pré-escolares de 5 a 6 anos}

\author{
Michael David Peraza Rodríguez \\ perazamichael92@gmail.com \\ ORCID: 0000-0002-5870-2203
}

Instituto de Altos Estudios “Dr. Arnoldo Gabaldon”, Venezuela

\begin{abstract}
RESUMEN
Introducción: La escabiosis constituye un problema de salud pública en especial en los niños, es una infestación cutánea, altamente pruriginosa y contagiosa endémica en países en vías de desarrollo, afecta a todos los grupos socioeconómicos y a todas las edades es causa la alta morbilidad Objetivo: Determinar la información sobre prevención de escabiosis que poseen los representantes de preescolares de 5-6 años de la comunidad de la Peña posterior a una intervención educativa Materiales y métodos: Investigación cuasi experimental de pre test y post test de las intervenciones educativas. El universo estuvo conformado por 143 representantes de preescolares, la muestra intencionada fue de 86 personas, el instrumento fue un cuestionario conformado por ítems de respuestas dicotómicas que se administró a los representantes antes de la intervención educativa y posterior a la misma. Resultados: Antes de la intervención existía muy baja información acerca de la prevención de la escabiosis lo que representaba el $67.4 \%$. Posterior a la misma se incrementa el número de representantes que mejoran su nivel de información hasta llegar a 73.2\%. Conclusión: El desarrollo y aplicación de la intervención educativa logro elevar los conocimientos sobre que es la escabiosis identificar cuáles son los factores de riesgo.
\end{abstract}

Palabras clave: Educación escabiosis; comunidad; enfermedades infecciosas
MP: Médico general integral egresado de la Universidad Nacional Experimental "Francisco de Miranda". Postgrado en medicina general integral, en la Universidad de la Ciencias de la Salud. Médico general tipo I del CDI "Cesar Augusto Ríos", Venezuela..

\begin{abstract}
Introduction: Scabies is a public health problem, especially in children. It is a highly pruritic and contagious skin infestation endemic in developing countries. It affects all socioeconomic groups and causes high morbidity at all ages. Objective: To determine the information on the prevention of scabies that the representatives of preschool children aged 5-6 years from the Peña community have after an educational intervention. Materials and methods: Quasiexperimental pre-test and post-test research of educational interventions. The universe was made up of 143 preschool representatives, the intended sample was 86 people, and the instrument was a questionnaire made up of items of dichotomous responses that were administered to the representatives before and after the educational intervention. Results: Before the intervention, there was very little information about the prevention of scabies, which represented $67.4 \%$. After the same, the number of representatives that improve their level of information increases to $73.2 \%$. Conclusion: The development and application of the educational intervention managed to raise the knowledge about what scabies is, to identify which are the risk factors.
\end{abstract}

Key words: Education; scabies; community; infectious diseases 
MP: Médico general integral egresado de la Universidad Nacional Experimental "Francisco de Miranda". Postgrado en medicina general integral, en la Universidad de la Ciencias de la Salud. Médico general tipo I del CDI "Cesar Augusto Ríos", Venezuela..

\section{RESUMO}

Introdução: A escabiose constitui um problema de saúde pública principalmente em crianças, é uma infestação cutânea altamente pruriginosa e contagiosa, endêmica em países em desenvolvimento, afeta todos os grupos socioeconômicos e é causa de alta morbidade em todas as idades Objetivo: Determinar as informações sobre prevenção de sarna que os representantes de crianças pré-escolares de 5-6 anos da comunidade La Peña têm após uma intervenção educativa Materiais e métodos: Pesquisa quase experimental de pré-teste e pós-teste de intervenções educativas. O universo foi constituído por 143 representantes da pré-escola, a amostra pretendida foi de 86 pessoas, o instrumento foi um questionário composto por itens de resposta dicotômicos que foi aplicado aos representantes antes e após a intervenção educativa. Resultados: Antes da intervenção havia muito pouca informação sobre a prevenção da escabiose, o que representava $67,4 \%$. Depois disso, o número de representantes que melhoraram seu nível de informação aumentou para $73,2 \%$. Conclusão: O desenvolvimento e aplicação da intervenção educativa foi capaz de aumentar o conhecimento sobre o que é sarna para identificar quais são os fatores de risco.

Palabras clave: Educação da escabiose; comunidade; doenças infecciosas

\section{INTRODUCCIÓN}

$\mathrm{L}$ as enfermedades infecciosas producidas por ectoparásitos constituyen un problema de salud pública en regiones de Asia, África, América Central y América del Sur donde ocasionan altas tasas de morbilidad, entre ellas la escabiosis o sarna es una patología de distribución cosmopolita, pudiendo presentarse de forma endémica y epidémica y afecta anualmente a unos 300 millones de personas y está íntimamente relacionada a la pobreza, falta de higiene y aglomeraciones por guerras y crisis económicas, afecta a personas de todos los niveles socioeconómicos sin distinción de edad, sexo, raza (1).

En Estados Unidos, durante el período 1937-1983, se presentó variaciones en su incidencia, de $5,4 \%$ en $1945,0,0 \%$ en 1955 , $1,0 \% 1960,3,6 \%$ en 1979 , con una reducción en los años posteriores. En Chile, estudios realizados en 1975 y 1981 demostraron una prevalencia en la ciudad de
Santiago de 5,0\% y 3,0\%, respectivamente. Estudios efectuados entre 1981 y 1987 en escolares de procedencia suburbana y rural de la $\mathrm{V}$ Región del país, demostraron una prevalencia de 0,6 a 24\%, con mayor predominio en el sexo femenino (2-3).

La prevalencia de la infestación en las comunidades varía y se ve influenciada por cambios en las condiciones socioeconómicas, migraciones, desplazamiento de poblaciones y situaciones de desastres naturales, que llevan a condiciones de hacinamiento, diagnóstico erróneo, tratamiento inadecuado y los posibles cambios del estado inmunitario de las personas (4).

La sarna o escabiosis es una dermatosis parasitaria producida por el ácaro Sarcoptes scabiei variedad hominis. La palabra proviene del término latino scabere que significa rasquido. La primera descripción la realizaron Bonomo y Cestón en el año 1647, quienes asociaron las lesiones cutáneas a la presencia del ácaro (5), es una enfermedad diseminada por todo el mundo, y debido a que se trata de una infestación, que 
usualmente no es notificada a las autoridades sanitarias y a menudo incorrectamente diagnosticada, su verdadera incidencia es desconocida (6).

El hombre desde el punto de vista epidemiológico, representa el su principal reservorio. Su mecanismo de transmisión es directo, por contacto entre persona a persona, lo que la hace una enfermedad de tipo familiar o de grupos cerrados. La posibilidad de contagio es de un $73-85 \%$, en condiciones higiénicas deficientes, por lo tanto, es importante considerar los factores de hacinamiento y promiscuidad. La transmisión a través de fómites es escasa (7). La higiene personal no necesariamente evita el contagio, pero puede variar la forma de presentación clínica, haciendo la enfermedad más leve y de difícil diagnóstico.

La edad es un factor que influye en la prevalencia de la escabiosis, pues se ha demostrado que es mayor en la infancia que en el adulto. Los ancianos asilados o en casa de reposo también tienen un aumento en su prevalencia. No hay diferencias por sexo. Se ha observado que la enfermedad aumenta en otoño e invierno, disminuyendo en primavera y verano. La tendencia al hacinamiento en invierno favorece su transmisión y a su vez, en verano las personas tienden a bañarse más, lo que elimina algunas formas juveniles de ácaros en la superficie de la piel, asociado a la mayor exposición a la luz ultravioleta, que tiene un efecto acaricida.

En Barquisimeto estado Lara, en el consultorio médico popular Dr. Che Guevara la Peña sector 1 al norte de la ciudad, asisten a las consultas representantes de preescolares de edades comprendidas entre 5-6 años con cuadros clínicos caracterizados por prurito intenso y erupciones pápulaeritematosas que se intensifican en horario nocturno, donde la prevalencia y la ausencia de estudios sobre los factores de riesgo que influyen en la presencia de esta patología.

La investigación planteada llena una necesidad existente de retroalimentación acerca de los factores de riesgo que intervienen en la aparición de la escabiosis en la comunidad, por lo que se planteó con objetivo determinar la información que poseen los representantes de los preescolares de la comunidad de la Peña evaluar antes y después de una intervención educativa.

\section{MATERIALES Y MÉTODOS}

I nvestigación cuasi experimental de pre test y post test de las intervenciones educativas, de tipo de intervención comunitaria. El universo estuvo conformado por 143 representantes de preescolares de la cual se tomó una muestra a 86 personas que representa el 60\% del universo; en la recolección de datos se utilizó como instrumento un cuestionario que se aplicó antes y después de la intervención educativa. La información obtenida fue tratada con estadística descriptiva. Estuvo guiada por las declaraciones de principios éticos internacionales, como fueron el código de Núremberg y la declaración de Helsinki. Para la realización del presente trabajo fue indispensable la anuencia de la dirección del centro donde se realizó el mismo. Por otra parte, a las personas objeto de estudio se le explicó detalladamente el objetivo de la investigación, puntualizándose que la información es estrictamente confidencial, y podían abandonarla en el momento que decidieran sin que ocasionara perjuicios en ningún sentido, se le solicito su consentimiento, el cual quedo firmado. 


\section{RESULTADOS Y DISCUSIÓN}

Tabla1. Nivel de conocimiento de los representantes de pre-escolares de la comunidad la peña sector I sobre la escabiosis y sus factores de riesgo antes y después de la intervención educativa.

\begin{tabular}{lccccc}
\hline \multicolumn{1}{c}{$\begin{array}{c}\text { Escabiosis y factores de } \\
\text { riesgo }\end{array}$} & \multicolumn{2}{c}{ f } & Antes & f & Después \\
\cline { 2 - 5 } & 56 & $65.1 \%$ & 20 & $17.2 \%$ \\
Inadecuado & 30 & $34.8 \%$ & 66 & $56.7 \%$ \\
Adecuado & & & & \\
\hline
\end{tabular}

En la Tabla 1 se observa las respuestas de los padres sobre escabiosis y sus factores de riesgo, tanto antes como después de la intervención educativa que el porcentaje de respuestas acertadas mejoro desde el $34.3 \%$ que tenían antes de la intervención hasta 56 $\%$ después de la intervención, no obstante no es lo deseable por cuanto un grupo importante aun después de la intervención educativa sigue respondiendo de forma incorrecta, con lo cual habría que plantearse el revisar la comprensión por parte de los representantes, evaluar si el nivel y complejidad como se transmitió la información es consonó con la formación de los padres etc.

En el proceso de la formación de la personalidad y de conductas sanas juega un papel fundamental la influencia de los padres y sus habilidades educativas El estilo educativo de los padres influye de forma decisiva en la adopción de hábitos saludables en los escolares que también deben ser acompañados de las acciones de cuidado en el hogar que propicien los padres para mantener la higiene y evitar infecciones como la escabiosis (8).

Tabla 2. Nivel de información sobre las medidas preventivas adecuadas para evitar el contagio de la escabiosis en pre-escolares de la comunidad la peña.

\begin{tabular}{lccccc}
\hline Medidas preventivas & & Antes & \multicolumn{3}{c}{ Después } \\
\hline Información insuficiente & 58 & $67.4 \%$ & 23 & $26.7 \%$ \\
Información eficiente & 28 & $32.5 \%$ & 63 & $73.2 \%$ \\
\hline
\end{tabular}

La Tabla 2 destaca que antes de la intervención existía baja información acerca de la prevención de la escabiosis lo que representaba el $67.4 \%$. Posterior a la misma se incrementa el número de representantes que mejoran su nivel de información hasta llegar a $73.2 \%$ no obstante continua la falta de información en un grupo que representa el $26,7 \%$. Con lo cual se hace necesario revisar las actividades desarrolladas y buscar nuevas alternativas. La educación para la salud es la estrategia fundamental de la promoción y la prevención de enfermedades, la transmisión de conocimientos a los padres favorece su parcelación en el desarrollo de una conducta encaminada a la conservación de la salud del niño, del 
colectivo y la sociedad. Para Martínez (9) durante la niñez, la referencia y apoyo principal recae en la figura paterna $\mathrm{y}$ materna ya que el niño precisa de una orientación constante debido a la inmadurez cognitiva de allí el rol fundamental que cumplen estos para favorecer hábitos saludables en el niño.

La educación es un fenómeno característico de la vida del ser humano, es una realidad social permanente, una función vital de la sociedad que ha acompañado al ser humano en el transcurso de su historia (8). De igual manera la salud y la enfermedad constituyen un proceso vital humano de carácter histórico, determinado social y económicamente y condicionado por las condiciones de vida, en la apropiación del espacio, del tiempo, de los bienes y servicios con que cuenta la familia.

Tabla 3. Evaluar el impacto de la información alcanzada en los representantes de preescolares de 5-6 años sobre la escabiosis sus factores de riesgo y su prevención.

\begin{tabular}{lccccc}
\multicolumn{1}{c}{$\begin{array}{c}\text { Nivel de impacto de la } \\
\text { intervención }\end{array}$} & f & \multicolumn{1}{c}{$\begin{array}{c}\text { Antes } \\
\text { \% }\end{array}$} & f & Después \\
\hline Adecuado & 26 & $30.2 \%$ & 65 & $75.5 \%$ \\
Inadecuado & 60 & $69.7 \%$ & 21 & $24.5 \%$ \\
Total & 86 & $100 \%$ & 86 & $100 \%$ \\
\hline
\end{tabular}

En la tabla 3 se observa un incremento del nivel de conocimientos de esta patología de los representantes posterior a la intervención desde un $69.7 \%$ de los representantes a un $75.5 \%$ logrando tener información adecuada sobre factores de riesgo y prevención. El hecho de que aun persista un grupo de padres con un nivel deficiente de información sobre la enfermedad, medidas de prevención y factores de riesgo es preocupante. La educación para la salud debe ser fundamentada en una pedagogía participativa y lograr no solo cambios cognoscitivos, sino cambios de conducta y estilos de vida, además comenzar desde edades tempranas pues el hábito desde pequeño hace más fácil llegar a la meta (8). Es más fácil formar actitudes positivas hacia la salud en un niño, que modificarlas en un adulto, probablemente los padres muestren resistencia a nuevos conocimientos, se hace necesario replantear acciones educativas.

El impacto que tienen los padres en la formación de los niños lo destaca la UNESCO (10) cuando señaló que los primeros educadores de los niños son los padres y madres, el espacio de aprendizaje por excelencia es el hogar, seguido del barrio, comuna y ciudad.

\section{CONCLUSIONES}

$\mathrm{E}$ l desarrollo y aplicación de la intervención educativa logro elevar los conocimientos sobre que es la escabiosis identificar cuáles son los factores de riesgo que contribuyen a desencadenar por lo que se considera esta investigación como adecuada en representantes de preescolares de 5-6 a los de la comunidad la peña sector , sin embargo se hace necesario continuar desarrollando actividades 
educativas dirigidas de forma permanente a los representes debido a que un grupo de la población estudiada continuo con déficit de conocimientos, con cual se requiere evaluar la efectividad del proceso educativo $\mathrm{y}$ plantearse nuevas estrategias.

Abordar la educación y formando hábitos, actitudes y valores es cómo se puede lograr que los niños y los jóvenes aborden la edad adulta con una efectiva cultura basada en la prevención y la salud. La educación para la salud es un proceso que acompaña toda la vida, que abarca las distintas etapas de la vida de las personas y que tiene como finalidad alcanzar el desarrollo y mantenimiento de un estado óptimo de salud.

Las prácticas de involucramiento de las familias en la educación para la salud deben partir desde el nivel preescolar y básico pues en los años tempranos las los padres y niños aprenden a respetarse y apoyarse mutuamente en las responsabilidades compartidas y de las alianzas tempranas permiten establecer modelos y relaciones que pueden favorecer el cultivo de hábitos saludables.

- Esta investigación fue realizada dentro de los estándares éticos, en pro de mejorar los procesos institucionales.

- No se requirió de ayuda financiera para su realización.

- La autora declara no poseer conflicto de interés alguno

\section{REFERENCIAS}

1. Pere L, Martínez W, Paradela S. Tratamiento de la escabiosis. Rev. Piel (Barc., Ed. impr.) 2011;26(2):95-102. doi: 10.1016/j.piel.2010.10.018.
2. Martorello S, Vanini M, Otero L. Escabiosis: una enfermedad que revela relaciones de poder en el encierro. Argentina. Universidad de La Plata; 2016.

3. Salazar D, Bejarano L. Identificación del Ácaro Sarcoptes Scabiei V. Hominis y su Relación. Con la Escabiosis. Ecuador. Universidad. $2016 . \quad$ Disponible en:https://repositorio.uta.edu.ec/jspu i/handle/123456789/19966

4. Maestre M, Martínez F, Fuentes $M$, Gómez R, et al, ¿Qué hacemos ante un brote de escabiosis en un hospital? Med Segur Trab (Internet) 2014; 60 (234) 239-243. Disponible en: http://scielo.isciii.es/pdf/mesetra/v6 0n234/caso.pdf

5. Hermida C, Vacas Prevención y tratamiento de escabiosis en los usuarios del Hospital Raúl Maldonado Mejía del Cantón Cayambe asociando el uso de Medicina Occidental con Medicina Tradicional y Componente Comunitario. Quito. Universidad San Francisco de Quito; 2012. Disponible en:

http://repositorio.usfq.edu.ec/handle /23000/5100

6. Campillos M, Causín E, Duro S, Agudo M, Martínez J. Escabiosis: revisión y actualización. MEDIFAM 2002; 12(7): 442-452. Disponible en: http://scielo.isciii.es/pdf/medif/v12n 7/hablemos.PDF

7. Rodríguez-Zúñiga $\mathrm{M}$, Torres-Panduro N. Escabiosis en Lima y Callao, 2016. Acta méd. Perú (Internet). 2017; 34(4): 335-336. Disponible en: http://www.scielo.org.pe/scielo.php? script=sci_arttext\&pid=S172859172017000400014\&lng=es. 
8. Mejía J. Educación para la salud en el mundo laboral, los servicios de prevención como motor de cambio para alcanzar un modelo óptimo de salud en el trabajo. España. Universidad de Sevilla; 2015. Disponible en:https://idus.us.es/bitstream/handl e/11441/40256/tesis\%20juan\%20ca rlos.pdf; jsessionid=6D2271EC6B8AE5F1D8D6 9FB49BE346EF? sequence $=1$

9. Martinez A, piqueras A. Importancia de los amigos y los padres en la salud y el rendimiento escolar. Electronic Journal of Research in Educational
Psychology.2010;8(1). Disponible en: 111-138.

http://repositorio.ual.es/bitstream/h andle/10835/793/Art_20_372.pdf?se quence $=1$

10. UNESCO. Participación familiar en la educación infantil latinoamericana. Santiago de Chile: Oficina Regional para la educación de América Latina y el Caribe/UNESCO. 2004. Disponible en:

https://unesdoc.unesco.org/ark:/482 23/pf0000139030 